\title{
Persistence of right umbilical vein: a singular case
}

\author{
Giovanni Pinna ${ }^{1, *}$, , Maria Pia De Carolis ${ }^{1, *}$, , Antonio Lanzone ${ }^{1 \oplus}$, \\ Gelsomina Del Sordo ${ }^{1 \oplus}$, Angela Botta ${ }^{2 \oplus}$, Sara De Carolis ${ }^{2 \odot}$ \\ Departments of ${ }^{2}$ Obstetrics, Gynaecology and ${ }^{1}$ Pediatrics, Fondazione Policlinico Gemelli IRCCS, Università Cattolica del Sacro Cuore, \\ Largo Francesco Vito 1, 00168 Rome, Italy.
}

\begin{abstract}
Background. Persistent right umbilical vein (PRUV) is one of the most common prenatally detected venous anomaly. In the intrahepatic variant (I-PRUV) the right umbilical vein fuses with right portal vein and through the ductus venous drains into inferior vena cava, while in the uncommon extrahepatic variant (E-PRUV), the vein bypasses the liver completely. E-PRUV has a worse prognosis compared to I-PRUV, due either to severe hemodynamic effects or to the frequent association with other severe fetal malformations.

Case. Here we report a case of E-PRUV with good outcome. Prenatal fetal ultrasonography (US) performed at 33 weeks of gestation in 28-year old woman, highlights the presence of E-PRUV with right UV draining in inferior vena cava. In the male neonate born at 35 weeks of gestation by C-section, the Apgar Score was $9^{5 \prime}-10^{10}$ and no other associated malformations and hemodynamic decompensation were found. Postnatal abdominal US showed the presence of enlarged paraumbilical veins.
\end{abstract}

Conclusions. The association of E-PRUV draining into the inferior vena cava with shunt through paraumbilical veins, could have preserved offspring by severe cardiac overload, positively affecting prognosis.

Key words: persistent right umbilical vein, fetus, neonate.

Persistent right umbilical vein (PRUV) is one of the most common prenatally detected venous anomaly, with an estimated wide range prevalence between 1 in 250 and 1 in $1250 .{ }^{1}$ In a recent retrospective review of 20,452 fetuses of consecutive pregnancies PRUV was identified in 23 cases, yielding an incidence of 1 in 889 total births $(0.11 \%){ }^{3}$

Two main presentations are described: Intrahepatic PRUV (I-PRUV) and Extra-hepatic PRUV (E-PRUV). The former is the most prevalent, since it is reported in about $90-95 \%$ of cases.,3 It may coexist with the left umbilical vein (UV) as an intrahepatic supernumerary structure or may present as unique vein joining the portal system at the level of the sinus venosus

Sara De Carolis

sara.decarolis@unicatt.it

*Giovanni Pinna and Maria Pia De Carolis are co-authors.

Received 24th October 2018, revised 26th January 2019, accepted 8th April 2019. and giving rise to the ductus venosus (DV). It is associated with normal DV development and its prognosis is good. ${ }^{4}$

E-PRUV is characterized by the persistence of right umbilical vein bypassing the liver and draining directly into right atrium or in intracardiac portion of inferior vena cava (IVC), or into iliac veins, subsequently these portosystemic anastomosis can cause cardiac overload and consequently fetal congestive heart failure. E-PRUV has a worse prognosis compared to I-PRUV due either to severe hemodynamic effects or to the frequent association with DV agenesis and other severe fetal malformations. ${ }^{2}$

We report a singular case of E-PRUV without DV and with the presence of enlarged paraumbilical veins (PUVs), with good prognosis.

\section{Case Report}

A 24-years-old Chinese woman, gravida 1, para 0 , was referred to our hospital at 33 weeks of 
gestation for vaginal bleeding due to central placenta praevia. The patients underwent prenatal diagnosis by amniocentesis that excluded chromosomal abnormalities.

FetalEchocardiography, performed atadmission in our hospital, showed moderate cardiomegaly with cardio-toracic circumference ratio equal to 0.62 (normal value < 0.50 ) and overload of right-sided heart, associated with ectasia of IVC and undetectable DV. For this reason, the scanning of the venous system, including the imaging of target vessels with two-dimensional colour Doppler mapping, was performed and showed that the UV ran parallel to the stomach and its intrahepatic course resulted medial with respect to the gallbladder. E-PRUV draining directly into IVC was diagnosed, due to this sonographic configuration and the initial signs of cardiac overload (Fig. 1).
Fetal ultrasonography (US) weekly performed confirmed this diagnosis, ruling out heart failure. At 36 weeks of gestation C-section was necessary due to further vaginal bleeding. At birth the neonate had a normal heart rate and the Apgar Score was of $9^{5}, 10^{10}$. The male neonate (weight $2500 \mathrm{~g}$, cranic circumference $32.5 \mathrm{~cm}$, length $48 \mathrm{~cm}$ ) was admitted to Neonatal Intensive Care Unit, and heart rate, $\mathrm{O}_{2}$ Saturation, blood pressure monitoring was started.

Echocardiographic evaluation, performed at one hour of life, showed normal cardiac structure and normal functional parameters. However, a mild dilatation of the right sections associated with moderate right ventricle hypertrophy and isosystemic pulmonary pressure were observed.

On the $2^{\text {nd }}$ day of life (DOL), the abdominal and cerebral US did not show any coexisting abnormalities, while Doppler US highlighted

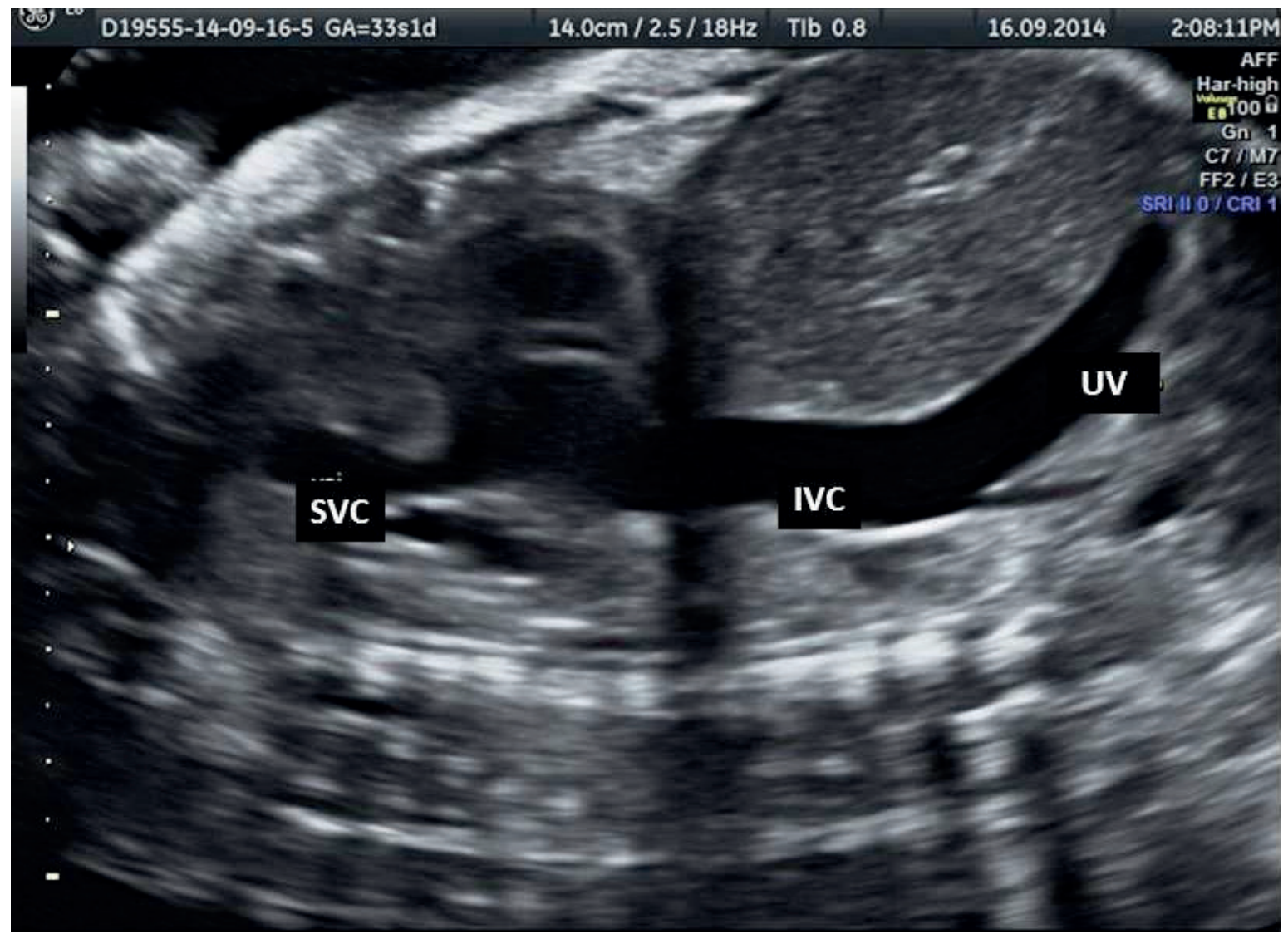

Fig. 1. Prenatal ultrasound examination shows the right umbilical vein (UV) draining directly in enlarged inferior vena cava (IVC). 
the portal vein joining to a patent venous paraumbilical circulation (Fig. 2). On the third DOL, an additional Echocardiographic evaluation confirmed the mild dilatation of the right-side sections while the pulmonary pressure was physiologically decreased. No indicative findings of liver dysfunction were observed, therefore on the sixth DOL the neonate was discharged.

At one month of life, the communication between portal vein and venous paraumbilical circulation was still present, but it was not detectable at 3 months of life. During this followup period no emerging clinical complications were detected.

Consent written informed consent was obtained from the patient's legal guardians for publication of this case report and any accompanying images.

\section{Discussion}

The incidence of umbilical vessels abnormalities is quite rare, but, in the last years, with the introduction of color Doppler and 3D scans, the diagnosis in utero of these conditions became more feasible and so more frequent. Anomalies of the umbilical and portal veins constitute the largest group of congenital venous anomalies detected in utero. They include three main entities: 1. agenesis of the DV with extrahepatic umbilico-systemic shunt or with intrahepatic umbilico-hepatic shunt; 2. PRUV with or without intact DV; 3 . UV varix. ${ }^{5}$ Among these, PRUV is the most frequently detected fetal venous system anomaly. In normal conditions, by the $4^{\text {th }}$ week of pregnancy the right UV begins to obliterate and on the $7^{\text {th }}$ week of gestation the process is completed. Failure of right UV regression results in the PRUV anomaly.

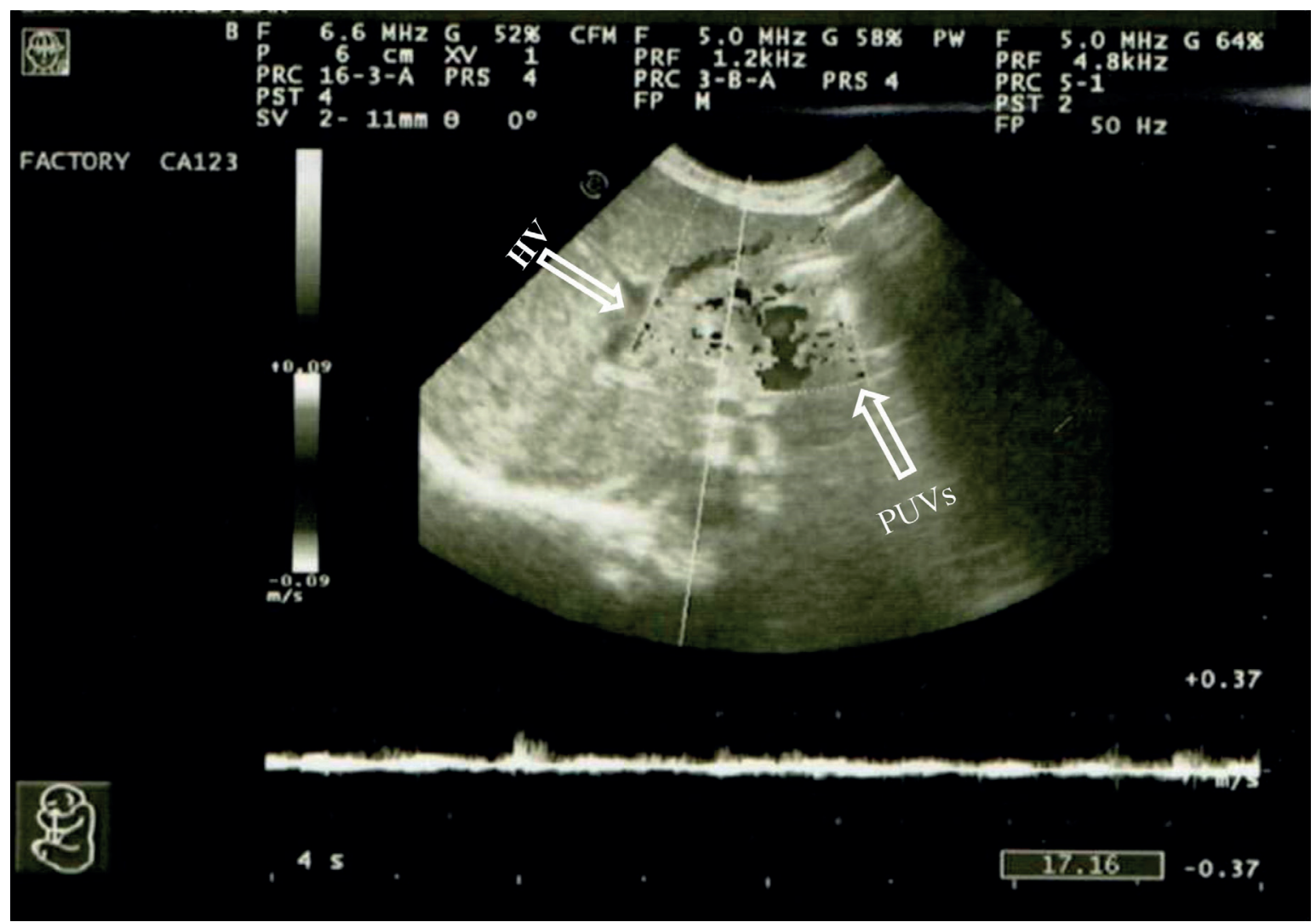

Fig. 2. Postnatal Doppler ultrasonography examination showing paraumbilical veins (PUVs) and hepatic vein (HV). 
The underlying pathogenetic mechanism causing PRUV is not completely clear. ${ }^{2}$ Teratogenic agents, such as retinoic acid or deficient folate ${ }^{6}$, as well as early obstruction of the left UV, caused by external pressure or thrombus occlusion, induced the PRUV anomaly. ${ }^{1}$ In fact, it has been suggested that primary or secondary occlusion by thromboembolic events, arising from the placenta, may lead to early streaming of blood through the right UV, therefore causing this anomaly. ${ }^{7}$

In our case, the images of prenatal and postnatal Doppler US suggest a possible role of an obstruction of umbilical vein flow at the level of the umbilical-portal junction, which is the area of "critical anastomosis" between left UV and omphalomesenteric veins. ${ }^{8}$ In prenatal Doppler US both left UV and DV were not detected. Since DV is the structure connecting left UV to VCI, an occlusion of left UV can reduce or interrupt the flow in $\mathrm{DV}$, therefore DV can result undetectable. On the other hand, the postnatal US showed the presence of dilated paraumbilical veins (PUVs). The PUVs are thick-walled with a similar structure to the umbilical vein. Together they constitute an accessory portal system which is confined between the layers of the falciform ligament and is in communication with the veins of the ventral abdominal wall. The constituents form an ascending series, namely Burow's veins, and Sappey's inferior and superior veins. The main channel of Sappey's inferior veins may be the remnant of the right umbilical vein since it communicates with the vessels of right rectus sheath and often communicates directly with the portal system within the right lobe of the liver. This channel communicates with the portal system in a variable manner: a) with the extra-hepatic part of the portal system at, or near, the recessus umbilicalis, b) directly with the intra-hepatic part by a branch to the free margin of the quadrate lobe, so forming an accessory portal system. ${ }^{9}$

Occlusion of left UV, due to thromboembolic event or to failure to delineate vascular connections ${ }^{10}$ before the right UV vanished, can determine a persistence of right UV, but also leads to shunting of umbilical blood through other vessels such as PUVs.

In our case the aforementioned pathogenetic hypothesis is also supported by normal karyotype and by the absence of other anomalies. In E-PRUV anomaly it is reported a frequent association with other congenital anomalies and chromosomal abnormality. It is generally recommended during prenatal US scan to evaluate the presence of other congenital abnormalities, such as genitourinary, gastrointestinal, cardiac and skeletal malformations. ${ }^{11,12}$

In our case, PRUV diagnosis was made by US and color Doppler according to criteria described by Jeanty ${ }^{7}$ : the UV ran right laterally respect to the gallbladder with an aberrant course toward the stomach (instead of being roughly parallel), and UV was not connected to the left portal vein. The extrahepatic variant was suspected in prenatal life because of the presence of PRUV draining directly into the IVC.

In previous reports, a high incidence of congestive heart failure, due to increased hemodynamic burden is clearly demonstrated, in case of extrahepatic drainage. In fact, the blood from the umbilical vein bypass the hepatic venous system flowing directly into the IVC and determining an overload of right-sided heart and heart failure. ${ }^{13}$

As underlined by Martinez ${ }^{1}$ and Hajdu ${ }^{14}$, E-PRUV requires prenatal careful monitoring to determine the timing of delivery in order to prevent congestive heart failure and hydrops. In our case, the diagnosis of E-PRUV occurred at 33 weeks of gestation, later than usual. ${ }^{1}$ After this time the fetus was weekly monitored to evaluate the impact of this condition on the hemodynamic system.

However, we did not observe a hemodynamic impairment during the time the mother was hospitalized probably due to the short interval between diagnosis and delivery. The C-section 
performed only two weeks after diagnosis could have influenced the hemodynamic outcomes, as well as the type of collateral channels opened. The presence of enlarged PUVs in postnatal Doppler US study can reflect the shunt by right UV through PUVs to the portal system and hence into the hepatic sinusoid. This umbilicoportalhepatic shunt decreases the cardiovascular overload determined by the direct connection of E-PRUV with IVC.

In conclusion, we report for the first time the association of E-PRUV with enlarged PUVs without severe hemodynamic effects, showing the possible association of E-PRUV with the intra-hepatic shunt.

In our opinion, in case of E-PRUV, it is important to define how blood drainage is established. The visualization of the connection of right UV to the systemic circulation as well as the presence of associated anomalies are the most important prognostic factors. In case of E-PRUV draining directly into the IVC or in the right atrium, there is an elevated risk of cardiac overload and hemodynamic stresses. Instead, when E-PRUV is associated with intrahepatic shunt, this risk could be attenuated. The accurate evaluation of this complex venous system during fetal life is necessary, even if difficult, to provide adequate antenatal counseling, timing of delivery and appropriate neonatal care.

\section{Acknowledgements}

We would like to thank the guardians for giving permission to submit this case report, which will undoubtedly develop our understanding about this rare condition.

\section{REFERENCES}

1. Martinez R, Gamez F, Bravo C, et al. Perinatal outcome after ultrasound prenatal diagnosis of persistent right umbilical vein. Eur J Obstet Gynecol Reprod Biol 2013; 168: 36-39.
2. Weichert J, Hartge D, Germer U, Axt-Fliedner R, Gembruch U. Persistent right umbilical vein: a prenatal condition worth mentioning? Ultrasound Obstet Gynecol 2011; 37: 543-548.

3. KumarSV, Chandra V, Balakrishnan B, Batra M, Kuriakose R, Kannoly G. A retrospective single centre review of the incidence and prognostic significance of persistent foetal right umbilical vein. J Obstet Gynaecol 2016; 36: 1050-1055.

4. Lide B, Lindsley W, Foster MJ, Hale R, Haeri S. Intrahepatic persistent right umbilical vein and associated outcomes: a systematic review of the literature. J Ultrasound Med 2016; 35: 1-5.

5. Yagel S, Kivilevitch Z, Cohen SM, et al. The fetal venous system, Part II: ultrasound evaluation of the fetus with congenital venous system malformation or developing circulatory compromise. Ultrasound Obstet Gynecol 2010; 36: 93-111.

6. Monie IW, Nelson MM, Evans HM. Persistent right umbilical vein as a result of vitamin deficiency during gestation. Circ Res 1957; 5: 187-190.

7. Jeanty P. Persistent right umbilical vein: an ominous prenatal finding? Radiology 1990; 177: 735-738.

8. White JJ, Brenner H, Avery ME. Umbilical vein collateral circulation: the caput medusae in a newborn infant. Pediatrics 1969; 43: 391-395.

9. Martin BF, Tudor RG. The umbilical and paraumbilical veins of man. J Anat 1980; 130(Pt 2): 305-322.

10. Jaiman S, Nalluri HB. Abnormal continuation of umbilical vein into extra-hepatic portal vein: Report of three cases. Congenit Anom (Kyoto) 2013; 53: 170175.

11. Hoehn T, Lueder M, Schmidt KG, Schaper J, Mayatepek E. Persistent right umbilical vein associated with complex congenital cardiac malformation. Am J Perinatol 2006; 23: 181-182.

12. Blazer S, Zimmer EZ, Bronshtein M. Persistent intrahepatic right umbilical vein in the fetus: a benign anatomic variant. Obstet Gynecol 2000; 95: 433-436.

13. Bell AD, Gerlis LM, Variend S. Persistent right umbilical vein case report and review of literature. Int J Cardiol 1986; 10: 167-175.

14. Hajdú J, Marton T, Kozsurek M, et al. Prenatal diagnosis of abnormal course of umbilical vein and absent ductus venosus--report of three cases. Fetal Diagn Ther 2008; 23: 136-139. 